\title{
TRANSFERENCIA LINGÜÍSTICA Y ALTERNANCIA DE CÓDIGO EN EXPRESIONES DE MANEJO DE GRUPO EN CLASES TIPO AICLE
}

\section{LANGUAGE TRANSFER AND CODE-SWITCHING IN CLASSROOM MANAGEMENT EXPRESSIONS IN A CLIL CONTEXT}

\author{
Erika Martínez Lugo \\ Universidad Autónoma de Baja California, Facultad de Idiomas
}

Donna Jackson-Maldonado

Universidad Autónoma de Querétaro, Facultad de Lenguas y Letras

Mónica Sanaphre Villanueva

Universidad Autónoma de Querétaro, Facultad de Lenguas y Letras

\section{RESUMEN}

Este estudio examina, describe y compara la alternancia de código $(A C)$ y la transferencia lingüística (TL) en emisiones de manejo de grupo de tres docentes en contexto de Aprendizaje Integrado de Contenidos y Lenguas Extranjeras (AICLE) en una ciudad del noroeste de México. En AICLE, la enseñanza de contenidos representa un reto para los docentes porque deben equilibrar el manejo de grupo, la enseñanza de los contenidos y, frecuentemente, el uso de su lengua extranjera. Los resultados muestran mayor presencia de tL que de AC. La tL es mayor en expresiones de orden directa y en expresiones instruccionales. Por su parte, la AC presentada es mínima, tan sólo seis casos, y es tanto del tipo intraoracional como interoracional.

Palabras ClaVe: adquisición de segundas lenguas; enseñanza del inglés; Aprendizaje Integrado de Contenidos y Lenguas Extranjeras; transferencia lingüística; alternancia de código 


\section{Abstract}

In this paper, we examine, describe and compare code-switching (CS) and language transfer (LT) in the speech of three teachers while managing the classroom in a Content and Language Integrated Learning (CLIL) context in a city in North Western Mexico. In the CLIL context, contents teaching is challenging for teachers as they have to balance managing the classroom, teaching contents and, often, the use of a foreign language. Our results show that teachers use LT more often than CS. LT occurs more frequently in direct orders and instructional utterances. Cs was used in only six instances and was both intra and intersentential.

KeYwords: second language acquisition; English language teaching; Content and Language Integrated Language; language transfer; code-switching

Fecha de recepción del artículo: 21 de marzo de 2015

Fecha de recepción de la versión revisada: 24 de octubre de 2016

Fecha de aceptación: 6 de diciembre de 2016

La correspondencia relacionada con este artículo debe dirigirse a:

Erika Martínez Lugo

erika_mtzlugo@uabc.edu.mx

Estudios de Lingüística Aplicada, año 35, número 65, julio de 2017, pp. 83-102

Dor: 10.22201/enallt.01852647p.2017.65.729 


\section{INTRODUCCIÓN}

El interés por ser bilingüe se ha incrementado significativamente en el mundo debido a las ventajas económicas, personales y profesionales que supone serlo. En consecuencia, se presenta un crecimiento en la oferta de colegios bilingües con diferentes métodos de enseñanza y con una variedad de condiciones que afectan el nivel de bilingüismo que se logra. Dentro de estas condiciones se encuentran el número de horas de clase en la lengua meta, el nivel de competencia lingüística tanto del docente como de los estudiantes, el propio docente y los materiales, entre otros.

Ser bilingüe es tener acceso a dos o más códigos lingüísticos y poder usarlos de manera simultánea (Hamers \& Blanc, 2004; Wei \& Wu, 2009). Existen variables tales como la edad y el modo de adquisición, el tipo de situación y lugar de aprendizaje, el tiempo de exposición a la lengua, entre otras, que afectan la manera como se aprende la segunda lengua y el nivel de dominio que se alcanza (Wei, 2007).

La relevancia de las variables de edad y el modo de adquisición están ligadas al tiempo y al tipo de exposición a las lenguas e impactan el nivel de dominio de la lengua en aspectos que van desde el sintáctico y semántico hasta el pragmático y fonológico en un acento marcado (Brown, 2007). Asimismo, existen diferentes formas de aprender dos lenguas desde edades tempranas: por medio de la asistencia a escuelas bilingües, por el hecho de vivir en un país bilingüe o vivir en un país en el que se habla una lengua distinta a la de la familia, o bien, por tener padres cuyas nacionalidades y lenguas maternas son diferentes (Wei, 2007). Al respecto, hay estudios sobre la adquisición del lenguaje en contextos bilingües, sobre el aprendizaje en salones de L2 y sobre la interacción en el salón de clase (Ellis \& Shintani, 2014; Hamers \& Blanc, 2004; Mitchel \& Myles, 2004).

Dos fenómenos naturales en situaciones de contacto de lenguas son la alternancia de código (AC) y la transferencia lingüística (TL). La AC es una estrategia de comunicación en la cual se usan, de manera alterna, dos o más lenguas (Hamers \& Blanc, 2004). En contextos de Aprendizaje Integrado de Contenidos y Lenguas Extranjeras (AICLE) algunos estudios presentan esta estrategia como el uso de la L1 o incluso como translanguaging sin utilizar el término AC (Celaya \& Ruiz, 2010; Cross \& Gearon, 2013; Mehisto, Frigols \& Marsh, 2008) y 
reconocen que es un fenómeno recurrente en el aula, con funciones específicas como empatía, identificación, uso óptimo de tiempos, entre otros. En contextos AICLE el docente emplea su L2 para la impartición de clases de contenido y de lengua de una manera entrelazada (Coyle, Hood \& Marsh, 2010).

El segundo fenómeno, la TL, es la influencia resultante de las similitudes y diferencias entre la lengua materna (L1) y la lengua que se aprende (L2) (Odlin, 1989). Algunos estudios tratan la TL en diferentes niveles, desde el léxico y el morfosintáctico hasta el fonológico, considerando, generalmente, lo que hace el estudiante como resultado de la TL por instrucción del docente (Agustín, 2010; Odlin, 1989).

Por otra parte, hay pocos trabajos sobre la AC y la TL de los docentes en salones de AICLE (CLIL, Content and Language Integrated Learning en inglés). Este trabajo se centra precisamente en escuelas en contexto AICLE, pues en dichos contextos las clases son impartidas, generalmente, por docentes cuya L1 no es la lengua meta a estudiar (Cambridge English, 2016). Se trata, como ya se ha mencionado, de contextos donde se emplea la L2 para la impartición de clases de contenido y clases de lengua de una manera entrelazada (Coyle et al., 2010), es decir, que en la clase converge la enseñanza de contenidos y la enseñanza de la lengua.

Llevar a cabo estudios respecto a la AC y la TL de los docentes en contextos AICLE podría arrojar información diversa para determinar si los fenómenos impactan en la producción de los niños por efecto de la enseñanza. También podría indicar aspectos a trabajar en la competencia lingüística de los docentes de dichos contextos. Asimismo, permitiría comprender que la AC y la TL son fenómenos comunes en el proceso de adquisición de segundas lenguas y que se presentan de manera natural en las personas bilingües.

En este trabajo se estudia la AC y la TL en emisiones de manejo de grupo por parte del docente en contextos AICLE a partir de las siguientes preguntas de investigación: ¿qué tipo de emisión de manejo de grupo presenta el docente en contexto AIClE?, ¿qué frecuencia tiene cada uno de estos tipos?, ¿cuál de ellos presenta mayor frecuencia de AC y TL? Asimismo, se determina si hay más AC que TL en las emisiones de manejo de grupo y si se usan emisiones de AC y TL de manera uniforme entre docentes. 


\section{Alternancia DE Código}

La AC es una estrategia de comunicación en la que se alterna el uso de dos o más lenguas en un mismo enunciado o conversación (Brice \& Brice, 2000; Grosjean, 1982; Hamers \& Blanc, 2004; Milroy \& Muysken, 1995; Poplack, 1980; Wei, 2007). Es un rasgo característico en el habla de las personas bilingües (Genesee \& Nicoladis, 2006) y no una deficiencia en la competencia lingüística de la persona en una, otra o ambas lenguas (Myers-Scotton, 1997; Wei, 2007). Se ha observado un alto grado de complejidad en la formulación de expresiones con AC en niños bilingües simultáneos comparado con la complejidad de AC que presentan los estudiantes de una lengua extranjera debido al tipo y tiempo de exposición al estudio de la lengua (Potowski, 2009). Esto porque la exposición y facilidad para utilizar la lengua en contexto no es la misma.

Grosjean (1982) indica que la presencia de AC está determinada por la persona con quien se interactúe. Es decir, cuando una persona bilingüe se encuentra con una persona monolingüe, utilizará la lengua materna que tenga el interlocutor, esta podrá ser su propia L1 o su L2, dependiendo del caso. Sin embargo, si se encuentra con otra persona bilingüe, la elección de la lengua a utilizar podrá ser su propia L1 o su L2 y, en ambos casos, podrá alternar el código o no (Figura 1).

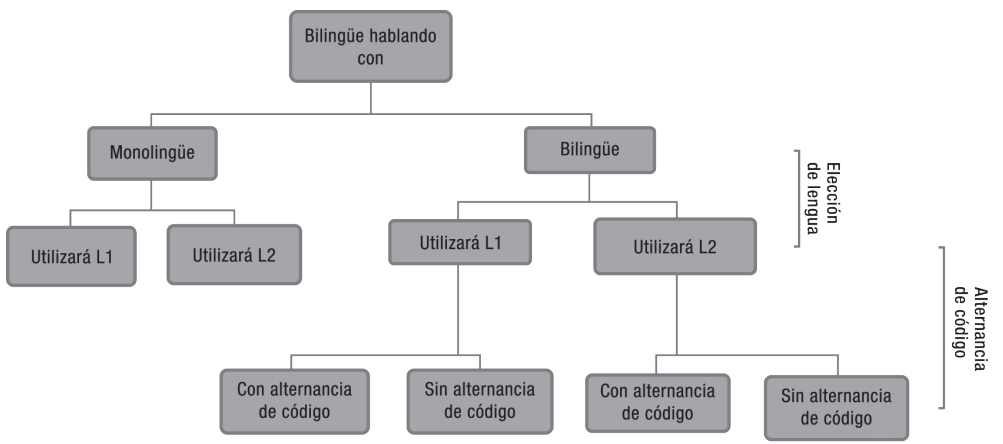

Figura 1. Elección de lengua y la alternancia de código (Adaptado de Grosjean, 1982:

129, en Wei, 2007; traducción de las autoras)

Poplack (1980) indica que la AC no se presenta en cualquier punto de la conversación y que está gobernada por reglas. Myers-Scotton (1997) propone el mo- 
delo del marco de la lengua matriz y el de la lengua incrustada en el que operan dos principios fundamentales. El primero, el principio del morfema de orden, indica que el orden de los morfemas en la locución resultante proviene de la lengua matriz. El segundo, el principio del morfema sistema, indica que la lengua matriz es el marco estructural del enunciado que resulte al hacer la AC.

Diversas investigaciones (Levine, 2009; Macaro, 2009; McMillan \& Turnbull, 2009; Nagy \& Robertson, 2009; Qing, 2010) indican que los docentes utilizan la AC como una estrategia de manejo de grupo que les permite un uso óptimo del tiempo, ya que pueden asegurar comprensión de contenidos de manera expedita, y es una estrategia para lograr metas específicas (Qing, 2010). Por otra parte, existe presión de los directores de las escuelas, padres de familia, estudiantes, así como de políticas gubernamentales para que, dentro del aula, todo sea hablado únicamente en la lengua meta. Sin embargo, se observa que la AC del profesor está generalmente motivada por el deseo de ser empático y solidario con sus estudiantes (Turnbull \& Dailey-O'Cain, 2009), por lo que es muy difícil cumplir con dicha disposición.

Ferguson (2009) agrega que es necesario estudiar la AC del docente en clases de contenido, pues es un fenómeno diferente al que se presenta en clases de lengua. En las primeras, lo primordial es que los estudiantes comprendan el contenido enseñado, por lo que la adquisición de lenguaje se convierte en un objetivo secundario. En las clases de lengua extranjera, por su parte, el objeto de estudio y el medio de instrucción son el mismo: la lengua meta.

La AC se puede presentar de manera intraoracional o interoracional (Saville-Troike, 2003) y en ambos casos puede ser léxica, morfosintáctica y fonológica. Los siguientes ejemplos, tomados de proyectos de estudiantes universitarios, muestran ambos tipos de AC:

a) Alternancia de código intraoracional: dentro de una misma oración como se observa en (1).

\section{(1) Ayer te miré in the mall.}

b) Alternancia de código interoracional: entre dos o más oraciones como en (2).

(2) Fuimos al centro comercial. There we bought the boots for the show. 
c) Alternancia de código léxica: el cambio por una palabra de L1 a L2 o viceversa, como se observa en (3).

(3) No sé cuál de ellos es su cousin.

d) Alternancia de código morfo-sintáctica: en la estructura de la oración de la L1 a L2. En (4) se aprecia la estructura del español y la alternancia del adjetivo en español:

(4) The house verde.

e) Alternancia de código morfo-fonológica: alternancia de sonidos para adaptarse a la L1 o L2 como en (5).

(5) Hoy tengo que mapear todo el piso de la casa. (Del inglés to mop /ma:p/ 'trapear' con el infinitivo del español trap-ear)

En este trabajo se considera la alternancia de código del tipo intraoracional e interoracional en emisiones de manejo de grupo.

\section{TRANSFERENCIA LINGÜÍSTICA}

La transferencia es un fenómeno de influencia interlingüística (Braga \& Domínguez, 2010; Jarvis \& Pavlenko, 2007; Ringbom, 2007). Por su parte, Sharwood y Kellerman (1986) indican que el término de influencia interlingüística permite incluir, bajo un encabezado, fenómenos tales como la transferencia lingüística, la interferencia, el escape, el préstamo y aspectos de pérdida de lenguaje relacionados con la L2.

La definición de transferencia lingüística de Odlin (1989) versa en el sentido de que la TL es la influencia de las similitudes y las diferencias entre la lengua materna y la lengua que se aprende. Grosjean (2011) considera además que la transferencia está relacionada con la competencia lingüística del individuo.

Odlin (1989) propone que la TL se presenta a nivel discursivo en la amabilidad (por ejemplo, para solicitar algo, ofrecer disculpas, ser directo o indirecto) y en la coherencia (respecto de la relevancia y la lógica), así como a niveles se- 
mántico (específicamente léxico), sintáctico (es decir, del orden en la estructura de la oración), fonético, fonológico y de la escritura. Agrega que factores no estructurales, como lo son la personalidad, factores socio-pedagógicos y el contexto social, así como la conciencia lingüística, entre otros, detonan la TL en el discurso de una persona. Odlin (1989) también asegura que la TL es un fenómeno que ocurre en todos los subsistemas lingüísticos.

Por su parte, MacWhinney (2004) expone en el modelo unificado de competencia que los aprendices de una L2 tienen como desafíos maximizar la TL positiva (cuando la estructura y el léxico coinciden), evitar la TL negativa (cuando dichas estructuras no coinciden, ocasionando, por ejemplo, falsos cognados) y sobreponerse a los efectos de la edad. Especifica que se pueden transferir elementos en el léxico, la fonología, la ortografía, la sintaxis y la pragmática, pero que rara vez se observarán en la morfología. Un ejemplo se muestra en la transferencia del género de los sustantivos de una lengua a los sustantivos de otra, como cuando en alemán en vez de usar la forma masculina der Mond, se produce incorrectamente la forma femenina die Mond, por influencia del español, donde luna lleva el género femenino.

Para los propósitos de este estudio la transferencia lingüística es la influencia que se detecta de la L1 en el uso de la L2. Se identifican los casos de TL independientemente del tipo y se analizan a partir de categorías de expresiones de manejo de grupo por parte de las docentes. Este estudio permite contrastar la TL con la AC.

\section{AprendizaJe Integrado de Contenidos y Lenguas EXTRANJeras}

El Aprendizaje Integrado de Contenidos y Lenguas Extranjeras (AICLE) es una forma de enseñanza en la que el contenido se presenta en la lengua meta. Es decir, en AICLE tanto en la enseñanza como en el aprendizaje, el contenido no es solamente el punto focal, también lo es la lengua meta. "Ambos [el contenido y la lengua] están entretejidos, aun cuando uno pueda tener mayor peso que otro en ciertos momentos" (Coyle et al., 2010: 1). Por ejemplo, en la clase de ciencia habrá momentos en que lo importante será el contenido respecto de las capas que tiene el planeta Tierra y su relevancia. En otros momentos lo será el estudio de la lengua inglesa, como sería, las partes de la oración.

Coyle, Hood y Marsh (2010) resaltan las múltiples ventajas que el AICLE trae consigo para los estudiantes, por ejemplo, los mantiene motivados, les ayu- 
da a desarrollarse cognitivamente y a mejorar sus habilidades orales. Consideran que el progreso en el manejo de lengua es mayor, además de que hay mayor input que se traduce en mayor output, los estudiantes aprenden a escribir y hablar en la lengua meta, así como a entender y crear significados en dicha lengua. El AICLE también proporciona ventajas en las escuelas que operan en dichos contextos, ya que alienta a estudiantes y docentes a lograr un mayor desarrollo e innovación mediante el uso de dos lenguas, un uso significativo que va más allá de la memorización. Los estudiantes en estos contextos tienen más práctica en la lengua meta por el tipo de proyectos que se manejan, como pueden ser presentaciones, exposiciones, entre otros, pero más que por la lengua en sí misma, por los contenidos que aprenden.

Asimismo, el AICLE es un motor que permite a los docentes involucrarse en el desarrollo curricular, en el diseño y en la elaboración de materiales. Les permite reflexionar sobre el proceso de aprendizaje en los estudiantes. Y, en el caso de los docentes no-nativo hablantes de la lengua meta, este contexto les ayuda a mejorar sus habilidades lingüísticas, por ejemplo, a adquirir mayor fluidez y a desarrollar un discurso más académico (Dale \& Tanner, 2012).

Sin embargo, el AICLE representa un reto para los docentes, ya que deben equilibrar el uso del tiempo, la distribución de actividades considerando la cantidad de estudiantes y de contenidos, así como atender la política institucional de utilizar únicamente la L2. Otro reto que se presenta en estos contextos es el manejo de grupo, pues el docente debe mantener buen control del mismo utilizando la lengua meta (y esta puede ser su L2). En este marco, es posible observar la presencia de otros fenómenos que también son asociados al bilingüismo como la AC y la TL (Odlin, 1989; Turnbull \& Dailey-O’Cain, 2009).

\section{MAneJo De GRUPo}

Harmer (2007) explica que, en una clase con buen manejo de grupo, el docente organiza eficazmente el espacio de trabajo, las actividades y las dinámicas de sus estudiantes y favorece el trabajo individual y en pequeños equipos sin perder el control del grupo. Agrega que la manera en que el docente se dirige a los estudiantes y el uso de la lengua materna, así como los momentos en los que se presenta, son elementos importantes para saber qué significado o usos tiene la L1 como elemento de manejo de grupo. 
En el manejo de grupo se observan detalles como cuánto se acerca o aleja físicamente el docente de los estudiantes, cómo modifica su tono de voz, cuántas veces habla (comparado con el número de intervenciones de sus estudiantes), cómo da instrucciones y cómo verifica la comprensión de las mismas. También qué tanto y qué tan bien planea su clase, cómo distribuye a los estudiantes en el aula y cómo se mueve dentro del salón de clase. Finalmente, también se consideran las expresiones que utiliza para manejar el grupo y qué tanto permite el uso de la L1 de los estudiantes.

\subsection{Categorías de manejo de grupo en este estudio}

Las categorías discursivas de manejo de grupo que se identificaron y estudiaron en este trabajo fueron de siete tipos: preguntas de comprensión de contenido académico, vocativos, órdenes directas, órdenes indirectas, motivadores, instruccionales e instruccionales en forma de pregunta. Estas categorías se presentan en el Cuadro 1.

Cuadro 1. Categorías de expresiones de manejo de grupo consideradas en este estudio

\begin{tabular}{|c|c|c|}
\hline CATEGoría dE EXPRESIONES DE MANEJO DE GRUPO & DESCRIPCIÓN & EJEMPLO \\
\hline $\begin{array}{l}\text { Preguntas de comprensión de } \\
\text { información (PCl) }\end{array}$ & $\begin{array}{l}\text { Preguntas que plantea la maestra para } \\
\text { verificar e indagar comprensión de } \\
\text { contenidos. }\end{array}$ & What is a noun? \\
\hline Vocativo (voc) & $\begin{array}{l}\text { Cuando se nombra al alumno para } \\
\text { llamar su atención. }\end{array}$ & $\begin{array}{l}\text { Daniela! } \\
\text { María! }\end{array}$ \\
\hline Orden directa (ORD) & $\begin{array}{l}\text { Orden directa relacionada con la } \\
\text { organización de la clase. }\end{array}$ & $\begin{array}{l}\text { Stop! } \\
\text { Sit down! }\end{array}$ \\
\hline Orden indirecta (ORD-IND) & $\begin{array}{l}\text { Orden indirecta por medio de una } \\
\text { aseveración. }\end{array}$ & I never said you could start. \\
\hline Motivadores (мот) & $\begin{array}{l}\text { Expresiones con las que la maestra } \\
\text { evalúa positivamente el esfuerzo, par- } \\
\text { ticipación o respuesta de los alumnos. }\end{array}$ & $\begin{array}{l}\text { Very good! } \\
\text { Excellent! }\end{array}$ \\
\hline $\begin{array}{l}\text { Instruccional (relacionada a la } \\
\text { instrucción de la clase) (INST) }\end{array}$ & $\begin{array}{l}\text { Expresiones relacionadas con la } \\
\text { organización de la clase pero que } \\
\text { no tienen que ver con el contenido } \\
\text { académico. }\end{array}$ & $\begin{array}{l}\text { I want you to bring that chart over } \\
\text { here. }\end{array}$ \\
\hline
\end{tabular}


Transferencia lingüística y alternancia de código en expresiones de manejo de grupo 93

Cuadro 1. Categorías de expresiones de manejo de grupo consideradas en este estudio (continuación)

\begin{tabular}{lll}
\hline CATEGORÍA DE EXPRESIONES DE MANEJO DE GRUPO & DESCRIPCIÓN & EJEMPLO \\
\hline \multirow{2}{*}{ Instruccional en pregunta (INST-P) } & Expresiones relacionadas con la & Why didn't you bring your pet? \\
& organización y orden de la clase & \\
& presentadas en forma de pregunta.
\end{tabular}

\section{Metodología}

\subsection{Participantes}

Las participantes en este estudio son tres docentes que imparten segundo, tercero y quinto grado, respectivamente, en la escuela primaria de contexto AICLE que nos permitió realizar tanto las observaciones como las grabaciones en audio de las clases. Las docentes son originarias de la misma ciudad fronteriza del noroeste de México donde se llevó a cabo el estudio y tienen contacto constante con la cultura del suroeste de los Estados Unidos a donde han viajado y vivido por periodos de uno a tres meses.

En el estudio se consideró la formación docente y la formación en la lengua meta de cada docente (Cuadro 2). La primera docente, P1, se formó como maestra en una escuela bilingüe y es licenciada en la enseñanza del idioma inglés. Contaba con cuatro años de experiencia docente al momento de realizar el estudio. $\mathrm{Su}$ formación en la lengua meta duró 13 años y fue en contexto AICLE.

La segunda docente, P2, tomó cursos de formación de la Secretaría de Educación Pública (SEP) y contaba con cuatro años de experiencia frente a grupo al momento de realizar el estudio. En cuanto a su formación en la lengua meta, cursó inglés por cuatro años en centros de idiomas de la ciudad fronteriza.

Finalmente, la tercera docente, P3, se formó como guía Montessori y contaba con siete años de experiencia frente a grupo, además de que su formación en la lengua meta tuvo lugar estudiando cuatro años en los Estados Unidos y uno más en un centro de idiomas en la ciudad donde se llevó a cabo este estudio.

Para determinar el nivel de dominio de la lengua meta (inglés para el caso) y de la competencia docente de las participantes se aplicó una prueba de autoevaluación que se adaptó de las pruebas de Gutiérrez-Clellen y Kreiter (2003) y Marian, Blumenfeld y Kaushanskaya (2007). Se optó por este instrumento debi- 
do a la practicidad de su aplicación como autoevaluación y porque al adaptarla se cubrió tanto el aspecto de la competencia lingüística como de la competencia docente. En el Cuadro 2 se presenta el resultado que cada una de las participantes obtuvo en la autoevaluación.

CuADRo 2. Descripción de las participantes

\begin{tabular}{|c|c|c|c|c|}
\hline $\begin{array}{l}\text { Participante / Grupo Que } \\
\text { IMPARTE }\end{array}$ & $\begin{array}{l}\text { FoRMACIÓN } \\
\text { DOCENTE }\end{array}$ & $\begin{array}{l}\text { FORMACIÓN } \\
\text { EN LA LENGUA META }\end{array}$ & $\begin{array}{l}\text { AutoEVALUACIÓN DE COM- } \\
\text { PETENCIA LINGÜISTICA }\end{array}$ & $\begin{array}{l}\text { EXPERIENCIA DOCENTE } \\
\text { EN AÑOS }\end{array}$ \\
\hline $\mathrm{P} 1 / 2^{\circ}$ grado & $\begin{array}{l}\text { Licenciatura en } \\
\text { docencia del idioma } \\
\text { inglés }\end{array}$ & $\begin{array}{l}13 \text { años de educación } \\
\text { bilingüe en México }\end{array}$ & 8.7 & 4 \\
\hline $\mathrm{P} 2 / 5^{\circ}$ grado & $\begin{array}{l}\text { Cursos de formación } \\
\text { docente SEP }\end{array}$ & $\begin{array}{l}4 \text { años de formación } \\
\text { en centro de idiomas } \\
\text { en México }\end{array}$ & 8.7 & 4 \\
\hline $\mathrm{P} 3 / 3^{\circ}$ grado & Guía Montessori & $\begin{array}{l}4 \text { años de educación } \\
\text { monolingüe en inglés } \\
\text { en EUA y } 1 \text { año en } \\
\text { centro de idiomas en } \\
\text { México }\end{array}$ & 9.7 & 7 \\
\hline
\end{tabular}

\subsection{Procedimiento}

Se pidió a las docentes que prepararan clases con actividades en las que hubiera interacción entre ellas y sus estudiantes, procurando que no fuera solamente pregunta-respuesta cerrada y que se evitara un discurso muy controlado. A las docentes se les explicó que se trataría de medir la extensión en la producción de los niños con el objetivo de que no se preocuparan de ser evaluadas en su ejercicio docente. Las clases que se observaron fueron de matemáticas, ciencias, inglés y arte.

Se audiograbaron ocho clases en sesiones de 30 a 45 minutos de duración en promedio para los grupos de segundo, tercero y quinto grado, respectivamente. Se transcribieron y se analizaron las ocho grabaciones en Childes con el código @ s para AC como se indica en MacWhinney (2000) y se decidió utilizar [+ gram] para TL ya que no había una codificación indicada para analizar la TL. El corpus resultante consiste en cerca de cinco horas de discurso transcrito. Las transcripciones fueron revisadas por una experta en adquisición de segundas lenguas, bilingüe simultánea (inglés y español) e investigadora de bilingüismo, con doctorado 
en lingüística. Su participación permitió identificar varias de las emisiones con TL. Asimismo, se consultó a una tercera hablante, nativa del inglés con grado de maestría en lingüística aplicada para aclarar dudas y aumentar así la confiabilidad.

\subsection{Resultados}

En primera instancia se presentan los resultados en los que se identificó el tipo de emisiones de manejo de grupo producidas por las participantes en este estudio (Cuadro 3).

CuADro 3. Tipos de expresiones de control y manejo de grupo: incidencias y porcentajes sobre el total de emisiones de las participantes $(N=895)$

\begin{tabular}{llcc}
\hline $\begin{array}{l}\text { TIPO DE EXPRESIÓN DE MANEJO DE } \\
\text { GRUPO }\end{array}$ & \multicolumn{1}{c}{ EJEMPLO } & OCURRENCIAS & PoRCENTAJE \\
\hline PCI & What is a pronoun? & 120 & 13.40 \\
VOC & Daniela! & 70 & 2.82 \\
ORD & Read the words carefully! & 265 & 16.31 \\
ORD-IND & $\begin{array}{l}\text { You don't need a pen right } \\
\text { now. }\end{array}$ & 146 & 4.47 \\
MOT & Very good! & 40 & 14.41 \\
INST & $\begin{array}{l}\text { You are going to do on your } \\
\text { own from eight to number } \\
\text { fourteen. }\end{array}$ & 129 & 13.97 \\
\hline INST-P & What are you going to do? & 125 & \\
\hline
\end{tabular}

Nota: PCl equivale a preguntas de comprensión de información; voc, a vocativo; ORD, a orden directa; ORD-IND, a orden indirecta; м0T, a motivadores; INST, a instruccional, e INST-P, a instruccional en pregunta.

En el Cuadro 3 destacan, con mayor presencia, las órdenes directas con un $29.61 \%$, seguidas de las órdenes indirectas, que representan $16.31 \%$, las instruccionales con un $14.41 \%$ y las instruccionales en forma de pregunta con $13.97 \%$. Las preguntas de comprensión de información representan $13.40 \%$, los vocativos $7.82 \%$ y, finalmente, las emisiones motivadoras $4.47 \%$. 
En el Cuadro 4 se describen las emisiones de manejo de grupo en general y por cada una de las participantes en las que se identificó la TL y la AC. Se indica el número de incidencias y el porcentaje que representa cada tipo respecto del total de emisiones. Las emisiones de manejo de grupo con mayor frecuencia de TL son las órdenes directas con 25 casos equivalentes a un $2.79 \%$, seguidas de las emisiones instruccionales con 16 casos equivalentes a un 1.79\%. Las emisiones con menor presencia son las preguntas de comprensión con dos casos equivalentes a $0.22 \%$. La frecuencia de emisiones de manejo de grupo con TL no es muy elevada, dado que solo representa $6.70 \%$ del total de emisiones de las tres docentes.

Por otra parte, las emisiones de manejo de grupo con más casos de AC fueron las preguntas de comprensión de información y las órdenes con dos casos, equivalente a $0.22 \%$ cada uno. Esto es, cuatro de los seis únicos casos que se presentaron de AC. Los otros dos casos corresponden, uno del tipo de orden indirecta y el otro de instrucciones en pregunta, equivalente a $0.11 \%$ del total de emisiones, respectivamente (Cuadro 4).

CuADRo 4. Emisiones con transferencia lingüística y alternancia de código por participante

\begin{tabular}{|c|c|c|c|c|c|c|c|c|c|c|}
\hline \multirow{2}{*}{ TIPO DE EMISIÓN } & \multicolumn{6}{|c|}{ OCURRENCIAS POR PARTICIPANTE } & \multicolumn{2}{|c|}{ TOTAL TL } & \multicolumn{2}{|c|}{ TOTAL AC } \\
\hline & P1TL & P1AC & P2TL & P2AC & P3TL & P3AC & EMISIONES & $\%$ & EMISIONES & $\%$ \\
\hline $\mathrm{PCl}$ & 1 & 2 & 0 & 0 & 1 & 0 & 2 & 0.22 & 2 & 0.22 \\
\hline ORD & 13 & 1 & 3 & 1 & 9 & 0 & 25 & 2.79 & 2 & 0.22 \\
\hline ORD-IND & 6 & 1 & 0 & 0 & 2 & 0 & 8 & 0.9 & 1 & 0.11 \\
\hline INST & 6 & 0 & 2 & 0 & 8 & 0 & 16 & 1.79 & 0 & 0 \\
\hline INST-P & 5 & 0 & 3 & 1 & 1 & 0 & 9 & 1 & 1 & 0.11 \\
\hline $\begin{array}{l}\text { Total de } \\
\text { emisiones }\end{array}$ & 31 & 4 & 8 & 2 & 21 & 0 & 60 & 6.7 & 6 & 0.67 \\
\hline
\end{tabular}

Nota: $\mathrm{P}=$ participante; 1,2 y 3 = número de participante; $\mathrm{TL}=$ transferencia lingüística; $\mathrm{AC}=$ alternancia de código; $\mathrm{PCI}=$ preguntas de comprensión de información; ORD = orden directa; ORD-IND = orden indirecta; INST = instruccional, e INST-P = instruccional en pregunta.

Respecto de cada participante, en el Cuadro 4 se observa que la P1 muestra mayor número de incidencias de emisiones con TL con 31 ocurrencias equivalente 
a 3.46\% del total de emisiones de todas las docentes. La P3 tuvo 21 emisiones con TL equivalente a $2.34 \%$, mientras que la P2 solo presentó ocho ocurrencias de emisiones con TL, es decir, $0.89 \%$ Con relación a la AC, la P1 presentó mayor número de casos con cuatro ocurrencias, correspondiente a $0.45 \%$, comparado con la P2, que presentó dos casos equivalentes a $0.22 \%$ y la P3, que no presentó caso alguno de AC.

Por su parte, en la Figura 2 se puede observar la distribución de las emisiones con AC y con TL. Mientras que las primeras se presentaron en seis ocasiones, representando un $0.67 \%$ del total de las emisiones, las segundas se presentaron en 60 ocasiones, equivalentes a $6.70 \%$.

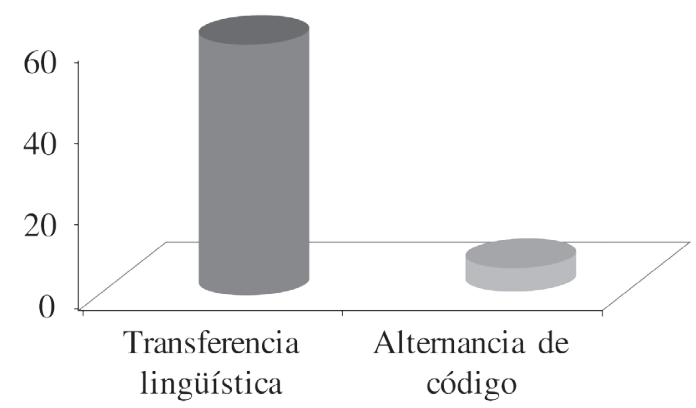

FIgURA 2. Ocurrencias con TL vs incidencias con AC

Por otra parte, la Figura 3 permite ver el comparativo entre las participantes del estudio y sus emisiones con TL y AC. Se aprecia que la P1 presenta la mayoría de emisiones tanto de TL como de AC (31 y cuatro, respectivamente) comparado con la P2 (ocho y 2) y la P3 (21 y cero).

Las tres participantes del estudio muestran más TL que AC, y es la P1 quien muestra un mayor número de ocurrencias tanto de AC como de TL. Por su parte, la P2 muestra menos TL que el resto de las participantes. Finalmente, la P3, que tiene mayor tiempo de experiencia con la lengua y frente a grupo, no presentó casos de AC, aunque sí de TL. Esto parece indicar que más tiempo de experiencia con la lengua no implica un mayor nivel de dominio en la lengua meta, y que tener más años de experiencia docente tampoco anula la presencia de TL. 


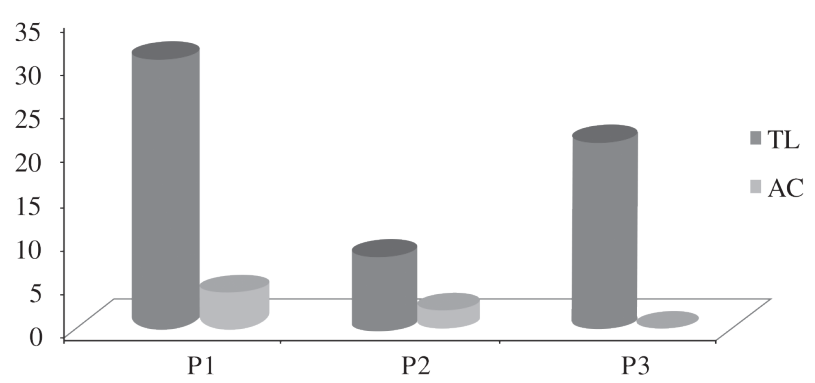

FIgURA 3. Comparativo de manejo de grupo con TL y AC por participante

Con respecto al tipo de Ac, se presentó un caso intraoracional de tipo léxico cuando la profesora verificaba comprensión gramatical, como se observa en (6). ${ }^{1}$

(6) D: Who can tell me what a naming part is?... ¿Qué es un naming part?... ¿Eddie?

Asimismo, se presentaron cinco casos de AC interoracionales con ideas completas como en los ejemplos (7) y (8).

(7) D: What do we need?... ¿Qué necesitamos para hacer una oración?

E: Naming part and action part.

(8) E: Teacher, ¿puedo ir a tomar agua?

D: No... No puedes ir a tomar agua... May I.

Finalmente, se puede observar que la incidencia de AC en este estudio no resultó alta, solamente seis casos, y que estos son para hacer un uso óptimo del tiempo y de los recursos en el aula.

\section{DiSCUSIÓN Y CONCLUSIONES}

Aunque existen estudios sobre la AC y sobre la TL, hacen falta estudios sobre ambos fenómenos en contextos AICLE en México, particularmente respecto del uso de la lengua meta por parte del docente. Este estudio explora, precisamente,

1 En este y los siguientes ejemplos, E se refiere a estudiante y D se refiere a docente. 
ambos fenómenos en dicho contexto. El colegio donde se llevó a cabo el estudio tiene una política de "solo inglés" por parte de docentes y estudiantes. Por esta política, aunada al hecho de que el conocimiento de dos lenguas suele asociarse a la AC, como lo establece Grosjean (1998), permitiría esperar una gran presencia de AC tanto en docentes como en estudiantes dentro de este contexto. Contrario a lo esperado, se encontró mayor cantidad de TL que de AC, probablemente como resultado del "efecto observador externo". Es decir, las docentes podrían no sentirse cómodas por estar en una situación de observación externa, lo que identificarían como una evaluación de su competencia lingüística. En estas situaciones, los docentes procuran utilizar un mínimo de palabras en español, lo que reduce su producción de AC y su uso de la lengua meta refleja TL.

Los resultados muestran que se presentó poca AC y que la presentada tiene una función principalmente como estrategia de manejo óptimo de tiempos. En la mayoría de los casos, esta se presentó cuando la docente utilizó su L1 (inglés) y cambió a español después de intentar obtener la información de los estudiantes sin éxito, por ejemplo: "You know how to say morado, I think" ('Creo que sabes cómo decir morado').

Asimismo, se observa en los resultados que el fenómeno que más se presenta es la TL y no la AC, que es más obvia al observador no-experimentado (aquel que no está pendiente o no sabe de las estructuras morfosintácticas, pronunciación, etc., al interactuar con otros), ya que es más identificable una palabra insertada en otra lengua (AC) que un cambio en la estructura (TL). Tal vez, debido a que es más sutil y difícil de identificar, la TL no ha sido descrita ampliamente en la bibliografía actual de AICLE en México. Otro resultado importante de la investigación es que se observaron diferencias en el uso de AC y TL dentro de las categorías de manejo de grupo. Específicamente, se identificó mayor frecuencia de TL en órdenes, tanto directas como indirectas.

También se puede observar que todas las participantes presentaron más TL que AC. Llama la atención el caso de la P1, que fue quien presentó un mayor número de ocurrencias de TL a pesar de ser la participante con más años de formación lingüística en escuela bilingüe y de tener una licenciatura en docencia del idioma inglés. Esta experiencia haría esperar que presentara menos TL y una influencia menor del español en sus producciones en la lengua meta.

No se puede concluir, entonces, que la formación o la experiencia lingüística o docente tengan influencia sobre la presencia de TL, sino que puede ser 
efecto de diferencias individuales. Un estudio con más participantes ayudaría a determinar con mayor precisión si la competencia lingüística y docente del profesor, así como el grado que imparte, son detonadores de la AC y la TL.

\section{REFERENCIAS}

Agustín Llach, María Pilar (2010). An overview of variables affecting lexical transfer in writing: A review study. International Journal of Linguistics, 2(1), 1-17. Recuperado de http:// www.macrothink.org/journal/index.php/ijl/article/view/445/pdf_2

Braga Riera, Jorge, \& Domínguez Romero, Elena (2010). Calque-free lectures? Spanish cross-linguistic influence in content teaching through English. ELIA: Estudios de Lingüística Inglesa Aplicada, 10, 113-135.

Brice, Alejandro, \& Brice, Roanne (2000). Language in the classroom: Comparisons of four bilingual environments. The South African Journal of Communication Disorders, 47, 91-98.

Brown, H. Douglas (2007). Teaching by principles: An interactive approach to language pedagogy (3a. ed.). White Plains: Pearson Longman.

Cambridge English (2016). tKT: CLIL Handbook for teachers. Cambridge: Cambridge University Press.

Celaya, María Luz, \& Ruiz de Zarobe, Yolanda (2010). First languages and age in Clil and non-CLIL context. International CLIL Research Journal, 1(3), 60-66. Recuperado de http:// www.icrj.eu/13-745

Coyle, Do; Hood, Philip, \& Marsh, David (2010). clil: Content and language integrated learning. Cambridge: Cambridge University Press.

Cross, Russell, \& Gearon, Margaret (2013). Research and evaluation of the Content and Language Integrated Learning (CLIL) approach to teaching and learning languages in Victorian Schools. Melbourne: The University of Melbourne.

Dale, Liz, \& TANner, Rosie (2012). clil activities: A resource for subject and language teachers. Cambridge: Cambridge University Press.

Ellis, ROD, \& Shintani, NATSuKo (2014). Exploring language pedagogy through second language acquisition research. Nueva York: Routledge.

Ferguson, Gibson (2009). What is next? Towards an agenda for classroom codeswitching research. International Journal of Bilingual Education and Bilingualism, 12(2), 231-241.

Genesee, Fred, \& Nicoladis, Elena (2006). Bilingual first language acquisition. En Erika Hoff \& Marilyn Shatz (Eds.), Blackwell handbook of language development (pp. 324-342). Oxford: Blackwell.

Grosjean, FrançoIs (1982). Life with two languages: An introduction to bilingualism. Cambridge: Harvard University Press. 
Grosjean, FrançOIs (1998). Transfer and language mode. Bilingualism: Language and Cognition, $1(3), 175-176$.

Grosjean, François (2007). The bilingual's language modes. En J. Nicol (Ed.). One mind, two languages: Bilingual language processing (pp. 1-22). Oxford: Blackwell.

Grosjean, François (2011). An attempt to isolate, and then differentiate, transfer and interference. International Journal of Bilingualism, 16(1), 11-21.

Gutiérrez-Clellen, Vera F., \& Kreiter, Jacqueline (2003). Understanding child bilingual acquisition using parent and teacher reports. Applied Psycholinguistics, 24(2), 267-288.

Hamers, Josiane F., \& Blanc, Michael H. A. (2004). Bilinguality and bilinguism (2a. ed.). Cambridge: Cambridge University Press.

Harmer, Jeremy (2007). How to teach English. Oxford: Pearsons Education.

Jarvis, Scott, \& Pavlenko, Aneta (2007). Crosslinguistic influence in language and cognition. Nueva York: Routledge.

LeVine, Glenn S. (2009). Building meaning through code choice in second language learner interaction: A D/discourse analysis and proposals for curriculum design and teaching. En Miles Turnbull \& Jennifer Dailey-O'Cain (Eds.), First language use in second and foreign language learning (pp. 145-162). Bristol: Multilingual Matters.

Macaro, Ernesto (2009). Teacher use of codeswitching in the second language classroom: Exploring 'optimal' use. En Miles Turnbull \& Jennifer Dailey-O’Cain (Eds.), First language use in second and foreign language learning (pp. 35-49). Bristol: Multilingual Matters.

MacWhinney, Brian (2000). The Childes project: Tools for analyzing talk. (3a. ed.). Mahwah: Lawrence Erlbaum. Recuperado de https://www.lti.cs.cmu.edu/projects/spoken-interfaces-and-dialogue-processing/childes-child-language-data-exchange-system

MacWhinney, Brian (2004). A unified model of language acquisition. En Judith F. Kroll \& Annette M. B. de Groot (Eds.), Handbook of bilingualism: Psycholinguistic approaches. Nueva York: Oxford University Press.

Marian, Viorica; Blumenfeld, Henrike K., \& Kaushanskaya, Margarita (2007). The language experience and proficiency questionnaire (LEAP-Q): Assessing language profiles in bilinguals and multilinguals. Journal of Speech, Language and Hearing Research, 50, 940-967. Recuperado de https://www.bilingualism.northwestern.edu/bilingualismpsycholinguistics/ files/MarianBlumenfeldKaushanskaya.pdf

McMillan, Brian, \& Turnbull, Miles (2009). Teachers' use of the first language in French immersion: Revisiting a core principle. En Miles Turnbull \& Jennifer Dailey-O’Cain (Eds.), First language use in second and foreign language learning (pp. 15-34). Bristol: Multilingual Matters. 
102 Erika Martínez Lugo, Donna Jackson-Maldonado \& Mónica Sanaphre Villanueva

Mehisto, Peter; Frigols, Maria Jesus, \& Marsh, David (2008). Uncovering CliL: Content and Language Integrated Learning in bilingual and multilingual education. Oxford: MacMillan Education.

Milroy, Lesley, \& Muysken, Pieter (1995). One speaker, two languages: Cross-disciplinary perspectives on code-switching. Cambridge: Cambridge University Press.

Mitchel, Rosamond, \& Myles, Florence (2004). Second language learning theories. Londres: Hodder Arnold.

Myers-Scotton, Carol (1997). Duelling languages: Grammatical structure in codeswitching. Nueva York: Oxford University Press.

Nagy, Krisztina, \& Robertson, Daniel (2009). Target language use in English classes in Hungarian primary schools. En Miles Turnbull \& Jennifer Dailey-O’Cain (Eds.), First language use in second and foreign language learning (pp. 66-86). Bristol: Multilingual Matters.

OdLIn, Terence (1989). Language transfer: Cross-linguistic influence in language learning. Cambridge: Cambridge University Press.

Poplack, Shana (1980). Sometimes I'll start a sentence in Spanish y termino en español: Toward a typology of code-switching. Linguistics, 18(7-8), 581-618.

Potowski, Kim (2009). Forms and functions of codeswitching by dual immersion students: A comparison of heritage speaker and L2 children. En Miles Turnbull \& Jennifer Dailey-O'Cain (Eds.), First language use in second and foreign language learning (pp. 87-114). Bristol: Multilingual Matters.

QING, XU (2010). To switch or not to switch: Examine the code-switching practices of teachers of non-English majors. Canadian Social Science, 6(4), 109-113.

Ringbom, HÅKan (2007). Cross-linguistic similarity in foreign language learning. Tonawanda: Multilingual Matters.

Saville-Troike, Muriel (2003). The Ethnography of Communication: An introduction (3a. ed.). Oxford: Blackwell.

Sharwood Smith, Michael, \& Kellerman, Eric (1986). Crosslinguistic influence in second language acquisition: An introduction. Londres: Pearson College Division.

Turnbull, Miles, \& Dailey-O’Cain, Jennifer (2009). First language use in second and foreign language learning. Bristol: Multilingual Matters.

WeI, Li (2007). The bilingualism reader (2a. ed). Nueva York: Routledge.

Wei, Li, \& Wu, Chao-Jung (2009). Polite Chinese children revisited creativity and the use of codeswitching in the Chinese complementary school classroom. International Journal of Bilingual Education and Bilingualism, 12(2), 193-211. 